1 Title Gelation of cassia gum by freezing and thawing

2

3 Authors Mika Iijima ${ }^{\mathrm{a}}$, TatsukoHatakeyama ${ }^{\mathrm{b}}$ and HyoeHatakeyama ${ }^{\mathrm{c} * *}$

4

5 Affiliation ${ }^{a}$ Faculty of Education, Nagasaki University, 1-14, Bunkyo-machi, Nagasaki, 852-8521, Japan

6

7

8

9

10

11

12

13

14

15

16

17

18

19

Total pages (including cover page and figures)

14

20

figure

8 


\section{Gelation of cassia gum by freezing and thawing}

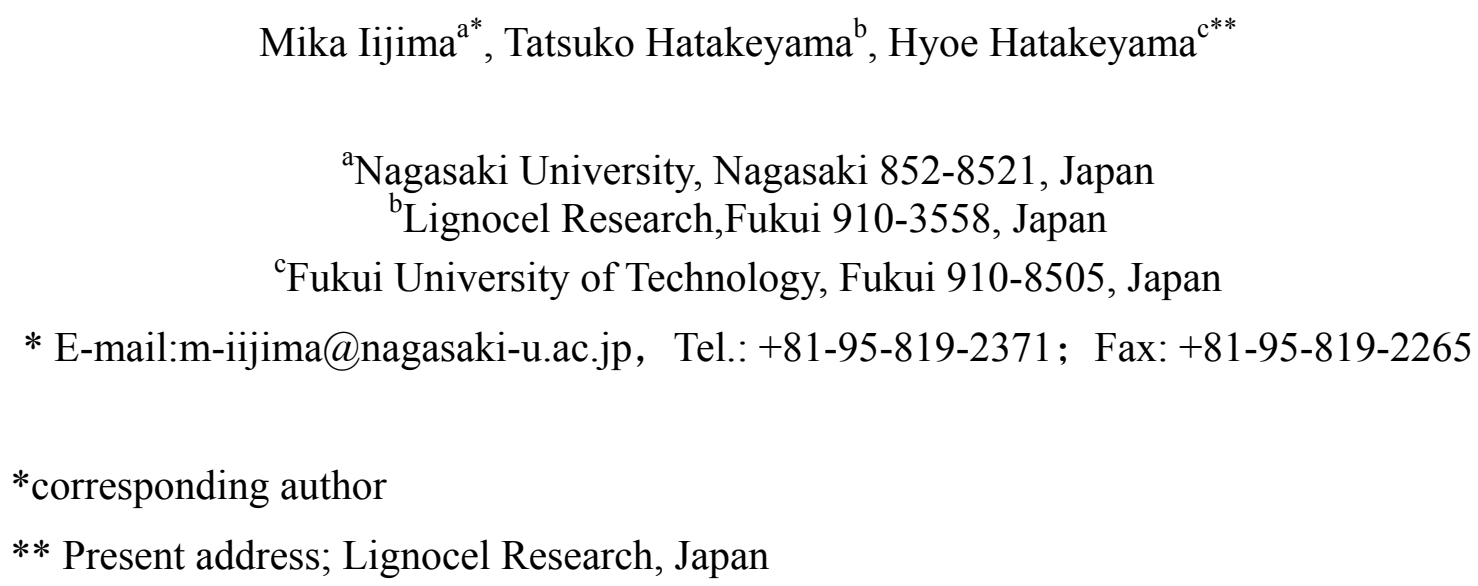

\section{Abstract}

Aqueous solution of cassia gum (CG), which is categorized as a galactomannan polysaccharide having mannose/galactose ratio $=5 / 1$, forms hydrogels by freezing and thawing. When frozen CG aqueous solution was thawed, transparent sol was separated from a turbid gel, i.e. syneresis occurred. Gel concentration (\{(Mass of dry gel) / (Mass of gel) \} 100) increased with increasing CG concentration. Viscoelastic properties of CG hydrogels formed by freezing and thawing were investigated by thermomechanical analysis (TMA) in water using an oscillation mode at $0.05 \mathrm{~Hz}$. Dynamic modulus ( $\left.E^{\prime}\right)$ increased from $3 \mathrm{kPa}$ to ca. $5 \mathrm{kPa}$ with increasing freezing rate. In contrast, $E$ ' maintained a constant value regardless of repeating number of freezing and thawing. From TMA results, it is concluded that the density of cross-linking network structure depends on the size of ice formed by freezing. At the same time,the low $E$ ' value of CG gels is ascribed to the fact that association of galactosyl side group is disturbed by the stiff chain attributed to the unsubstituted region of CG

Keywords; TMA, galactomannan polysaccharide, cassia gum, hydrogel, freezing and thawing 
57 Cassia gum (CG), locust bean gum (LBG), tara gum (Tara-G), guar gum (GG) and 58 fenugreek gum are categorized as galactomannan polysaccharides. Galactomannan 59 polysaccharide is extracted from plant seeds of various plants, i.e. CG is extracted from 60 plant seeds for Cassia tora, [1,2] LBG is Ceratoniasiliqua, Tara-G is 61 Caesalpiniaspinosa, GG is Cyamopsistetragonoloba and FG is 62 Trigonellafoenum-graecum. Galactomannan polysaccharides consist of a 1,4- $\beta$-D-mannose backbone and 1,6- $\alpha$-D-galactose side chains. A structural difference among the five gums is the mannose/galactose ratio ( $\mathrm{M} / \mathrm{G}$ ratio), which is $5,4,3,2$ and 1 for CG, LBG, Tara-G, GG and FG, respectively. GMs are widely used in food, pharmaceutical and cosmetic industries as stabilizers and emulsifiers since GMs hold a large amount of water [3]. Recently, the conformation of GM galactomannans with different mannose/galactose ratios has been investigated by the molecular modeling approach [4]. However, CG has received little attention sincetheeligibility has been recognized in recent years [5].

A large number of polysaccharides, such as agarose, $\kappa$-carrageenann andcurdlan, are known to form hydrogels when aqueous solution of the above polysaccharides is cooled, by which molecular chains of polysaccharides are co-aggregated and a three dimensional network structure is established. Among various types of gelation methods, such as, cooling, heating and ionic binding, gelation of polymers by freezing and thawing is not common. A rare example is LBG[6-9]. Among synthetic polymers, poly(vinyl alcohol)is only known to form hydrogel by freezing and thawing [10-13]. The above facts suggest that polymers having the capability of gel formation by freezing and thawing are rare. When the chemical structures of LBG and CG are compared, it is thought that CG may have the capability of gel formation by freezing and thawing.

In our previous studies, we employed thermomechanical analysis (TMA) to measure the viscoelastic properties of polysaccharide hydrogels in water [14-17]. Using the above technique, both static and dynamic mechanical properties of hydrogels at various temperatures can be measured by controlling water temperature [14]. In this study, gelation of CG by freezing and thawing is investigated by TMA in water. 


\section{Experimental}

\section{Sample preparation}

CG in powder form was provided by Kibun Food Chemifa Co. Ltd., Japan. The commercial name is Rheo Ranger TM SR. CG was solved in deionized water at $298 \mathrm{~K}$ to obtain $0.1 \sim 1.5 \%$ solutions. The solutions were annealed at $376 \mathrm{~K}$ for $2 \mathrm{~h}$. After annealing at $378 \mathrm{~K}, 5,10,20,30$ or $50 \mathrm{ml}$ of CG solutions were put in a $100 \mathrm{ml}$ polyethylene container $(49 \varphi \times 72 \mathrm{~mm})$. The CG solution in PE container was directly transferred to a freezer whose temperature was $253 \mathrm{~K}$ and maintained for approximately $18 \mathrm{~h}$. Cooling rate was measured by thermometer. Obtained cooling rates were 3.0, 2.5, $1.5,1.0$ and $0.7 \mathrm{~K} \mathrm{~min}^{-1}$, respectively. Frozen samples were thawed slowly at $298 \mathrm{~K}$. This process took more than $6 \mathrm{~h}$. The above freezing and thawing cycles are referred to as " $n$ ". After freezing and thawing, the samples were stored at $298 \mathrm{~K}$.

The gel and sol were centrifuged at $1000 \mathrm{rpm}$ for $5 \mathrm{~min}$. Gels were removed from the centrifugal vessel and weighed quickly. The gel ratio was calculated according to the following equation.

Gel ratio $\left(\mathrm{g} \mathrm{g}^{-1}\right)=($ Mass of gel $) /($ Mass of gel + mass of sol $)$

The gel or sol was removed from the container and dried at $393 \mathrm{~K}$ for $10 \mathrm{~h}$ in an oven and dry mass of the gel was recorded. Polymer concentration was calculated according to the following equation.

Polymer concentration $(\%)=\{($ Mass of dry gel $) /($ Mass of gel $)\} 100$

The mass of samples was measured using a Mettler Toledo microbalance (XS205). Precision was $\pm 1.0 \times 10^{-5} \mathrm{~g}$. Concentration ratio was calculated according to the following equation.

$$
\text { Concentration ratio }=\text { Polymer concentration } / \text { Sol concentration }
$$


An SII Nano Technology Inc. thermomechanical analyser (TMA, SII Nano Technology Inc. TMA/SS 150) equipped with a newly designed sample holder was used [14]. A quartz rod with uniform cross sectional area $\left(5.09 \times 10^{-5} \mathrm{~m}^{2}\right)$ was used as a probe. The sample holder was immersed in water whose temperature was controlled at $298 \mathrm{~K}$. The gel sample was placed in a quartz sample pan with diameter $9 \mathrm{~mm}$ (inner diameter) and height $5 \mathrm{~mm}$ and immersed in $70 \mathrm{ml}$ of water. The sample was compressed by quartz probe.

Dynamic measurements were carried out in water at $298 \mathrm{~K}$ using a quartz probe. Operating frequency was $0.05 \mathrm{~Hz}$. Measurements were carried out for $5 \mathrm{~min}$. The conditions employed were dynamic modulus $\left(E^{\prime}\right)$ and $\tan \delta$. From Lissajous diagram, $E^{\prime}$ and $\tan \delta$ were obtained. $1 \%$ CG hydrogel was used as a sample for TMA measurements.

\section{Results}

The chemical structure of $\mathrm{CG}$ is shown in Fig. 1.A turbid gel was formed by freezing-thawing process of $\mathrm{CG}$ aqueous solution, and transparent liquid was separated from the gel at the time of thawing, i.e. syneresis occurred. When this gel is treated at a

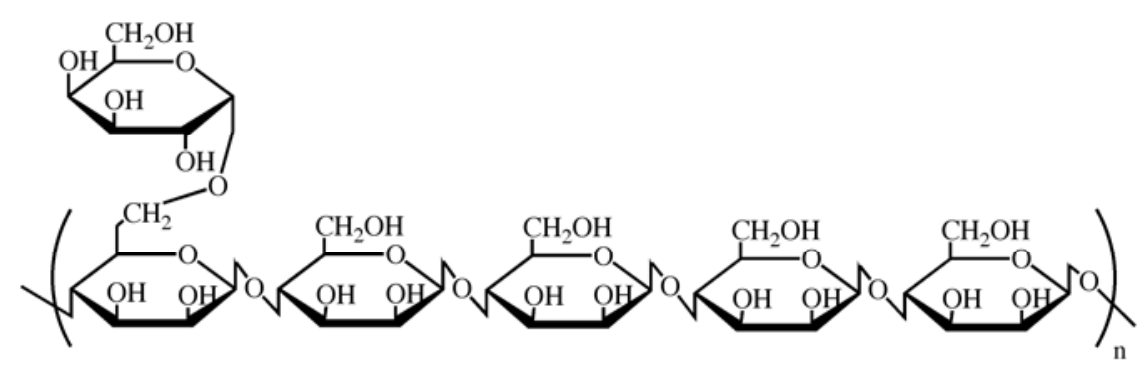

Fig. 1 Chemical structure of cassia gum 
Effect of sol concentration on gelation

Gelation by freezing and thawing is affected by various factors such as concentration of sol, freezing rate [9], number of freezing and thawing [7] and thermal history of sol state [8]. At first, in this study, effect of sol concentration on gelation was investigated. Fig. 2 shows relationships between polymer concentration in gel and sol concentration. Freezing rate was $2.5 \mathrm{~K} \mathrm{~min}^{-1}, n$ was 1 or 3 . The broken line shown in Fig. 2 indicates that polymer concentration is assumed to be the same as sol concentration. It is clearly seen that polymer concentration is far larger than that shown in the broken line. This indicates that $\mathrm{CG}$ markedly condensed during freezing process. As shown in Fig.2, polymer concentration increases with increasing sol concentration. It is considered that molecular co-aggregation occurs during freezing. CG concentration in the gel state obviously increases and an excess amount of water was excluded from the gel network.

4 
$0.1 \%$ sol is condensed ca. 35 times. Concentration ratio decreased with increasing sol concentration. This indicates that molecular co-aggregation is markedly observed when sol concentration is small.

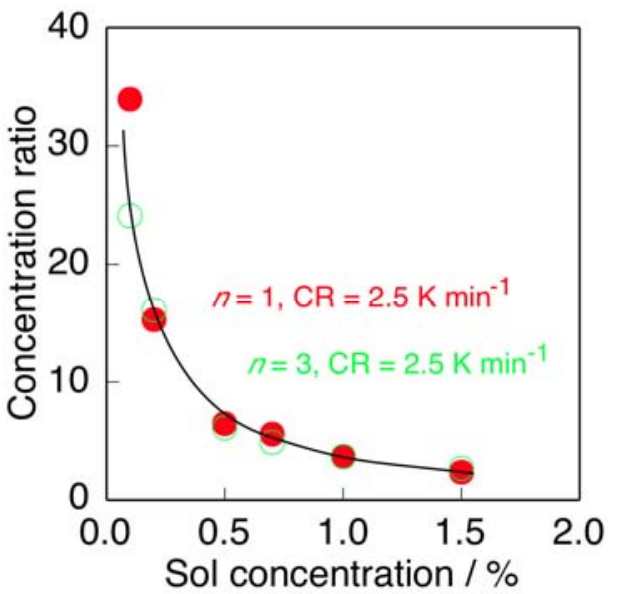

Fig.3 Relationship between concentration ratio and sol concentration.

Cooling rate $=2.5 \mathrm{~K} \mathrm{~min}^{-1}$, Closed circle; $n=1$, open circle; $n=3$

Effect of number of freezing and thawing $(n)$

As described in the experimental section, the number of freezing thawing varied from 1 to 10 times and gel concentration was measured. Fig.4 shows relationship between polymer concentration and $n$. The number of freezing and thawing scarcely affects the polymer concentration. This indicates that once the network structure is formed, molecular rearrangement does not occur. In contrast, it is clearly seen that cooling rate evidently affects polymer concentration, i.e. concentrations of gel formed at $1 \mathrm{~K}$ $\mathrm{min}^{-1}$ are larger those formed at $2.5 \mathrm{~K} \mathrm{~min}^{-1}$. Accordingly, in the latter sections of this paper, cooling rate was fixed at either or both $n=1$ and $n=3$. 


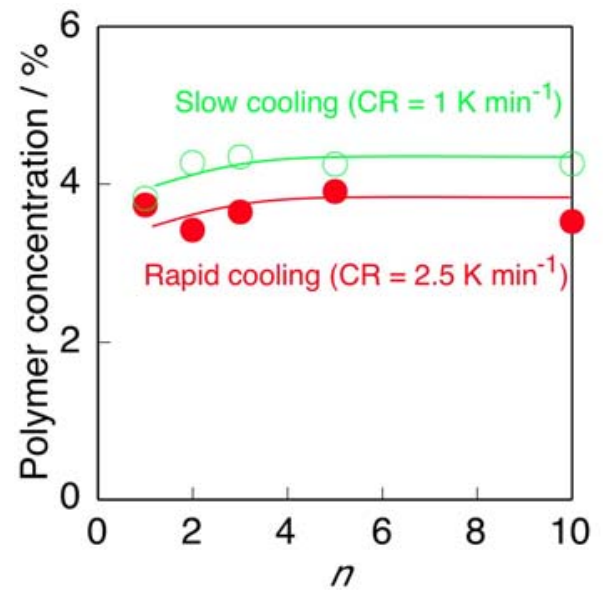

195

196

197

198

199

200

201

202

203

204

205

206

207

208

209

210

211

212

213

214

215

216

217

218

219

Fig.4 Relationship between polymer concentration and $n$.

Concentration $=1 \%$, Closed circle; $\mathrm{CR}=2.5 \mathrm{~K} \mathrm{~min}^{-1}$, open circle; $\mathrm{CR}=1 \mathrm{~K} \mathrm{~min}$

8

\section{Effect of cooling rate}

Fig. 5 shows polymer concentration formed at $n=1$ (Fig. 5 (a)) and formed at $n=3$ (Fig.5 (b))as a function of sol concentration. At rapid cooling $\left(2.5 \mathrm{~K} \mathrm{~min}^{-1}\right)$, polymer concentration increased with increasing sol concentration regardless of $n$. However, at slow cooling, polymer concentration maintains a constant value regardless of sol concentration. The effect of $n$ is slightly observed when cooling is $1.0 \mathrm{~K} \mathrm{~min}$. Concentrations of gel formed by $n=3$ at $1.0 \mathrm{~K} \mathrm{~min}^{-1}$ are higher than those by at $n=1$. In 8 contrast, no significant difference by changing $n$ was found for gels formed at $2.5 \mathrm{~K}$ $\mathrm{min}^{-1}$. Molecular co-aggregation during freezing depends on cooling rate. This indicates 0 that the size of ice affects the cross-linking zone formation.

11


(a)

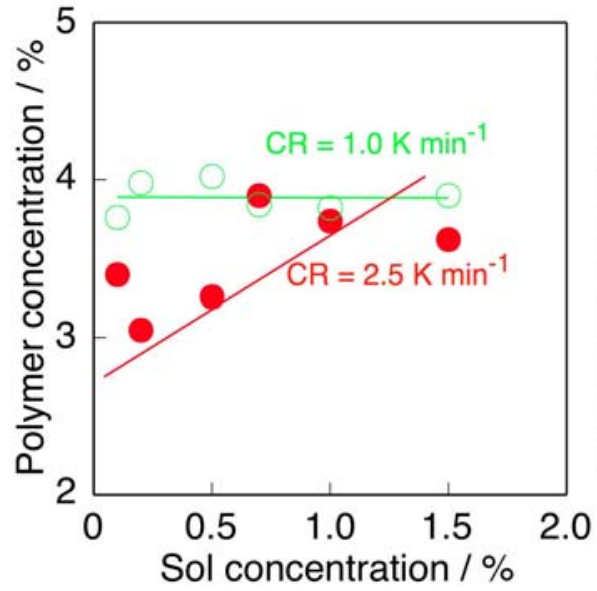

(b)

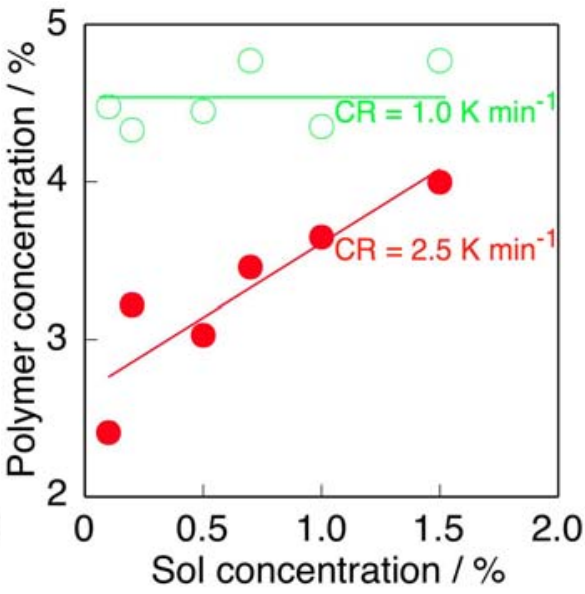

221

222

223

224

225

226

227

228

229

230

231

232

233

Fig.5 Relationship between polymer concentration and sol concentration.
(a) $n=1$, (b) $n=3$. Closed circle; $\mathrm{CR}=2.5 \mathrm{~K} \mathrm{~min}^{-1}$, open circle; $\mathrm{CR}=1 \mathrm{~K} \mathrm{~min}{ }^{-1}$

Cooling rate varied from 0.5 to $3 \mathrm{~K} \mathrm{~min}^{-1}$ and gel formation was examined. Fig.6 shows the relationship between polymer concentration and cooling rate. $n$ was found to have no effect on polymer concentration. Polymer concentration decreases with increasing cooling rate. It is evident that cooling rate is a major factor in controlling gel formation.

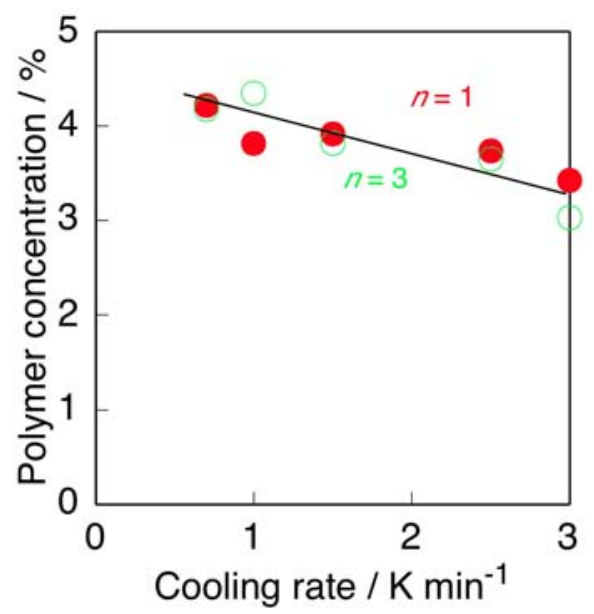

Fig.6Relationship between polymer concentration and cooling rate.

Sol concentration $=1 \%$, Closed circle; $n=1$, open circle; $n=3$ 
Representative gels which formed from sol concentration $=1 \%, n=1$, cooling rate $0.7 \sim 3.0 \mathrm{~K} \mathrm{~min}^{-1}$ were measured by TMA in water [9]. The sample was taken from different parts of the frozen gel and no significant difference was observed in the values of $E^{\prime}$ and $\tan \delta$. As reported in our previous papers, dynamic modulus $E$ ' was calculated 242 from Lissajous diagram [14]. Fig.7 shows the relationship between $E$ ' and cooling rate. $243 E^{\prime}$ increased from $3 \mathrm{kPa}$ to ca. $5 \mathrm{kPa}$ with increasing cooling rate. $E$ ' maintained a constant value regardless of $n$, although the figure is not shown. The cooling rate dependency of gelation is also confirmed by TMA results. A densely cross-linked network structure is formed by slow cooling.

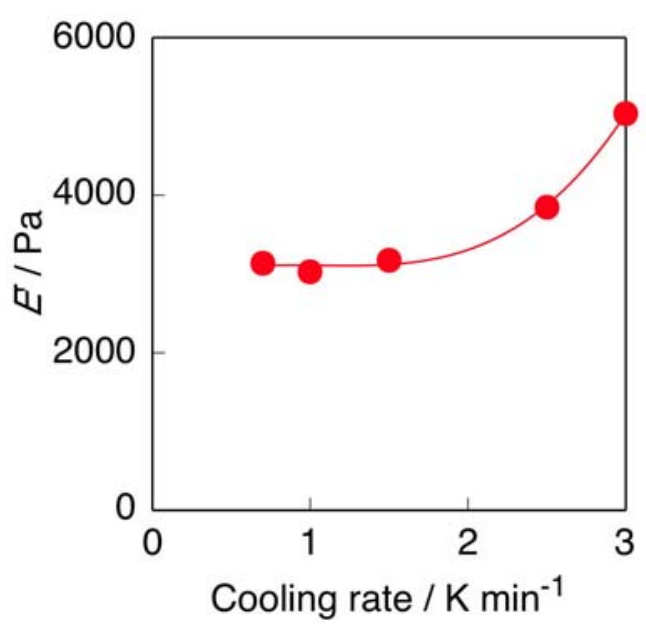

Fig.7 Relationship between $E$ ' and cooling rate.

Concentration $=1 \%, n=1$

\section{Discussion}

From the above results, it was confirmed that CG forms hydrogels by freezing and thawing. At the same time, it was found that gels are formed in a limited concentration range, from 0.1 to $1.5 \%$. Among a series of galactomannans, LBG is known to form hydrogel by freezing and thawing [6-9]. LBG forms hydrogels in a concentration from 0.5 to $10 \%$ under the same conditions in which CG hydrogel is 
262 formed in this study. Both hydrogels are turbid, however, when cooling rate is the same,

263 CG excludes a large amount of water from gel as shown in Fig. 2. In contrast, LBG 264 showed no syneresis when concentration exceeds $3 \%$ [9]. As shown in Fig. 4, $n$ 265 scarcely affects polymer concentration, although cooling rate dependency on the 266 gelation is noticeable. As shown in Fig. 6, polymer concentration decreases with 267 increasing cooling rate. Viscoelastic properties measured by TMA exhibit clearly the 268 characteristic features of CG gel. $E$ ' values of CG gels are compared with those of LBG 269 in Fig. 8. E' values of CG are far lower than those of LBG. When comparison is made 270 on a same scale of LBG, no marked change is observed for CG, when $n$ and cooling rate 271 are varied.

272 273 274

(a)

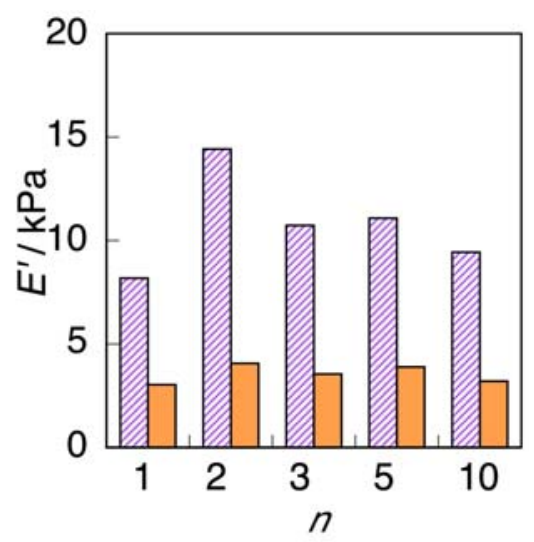

(b)

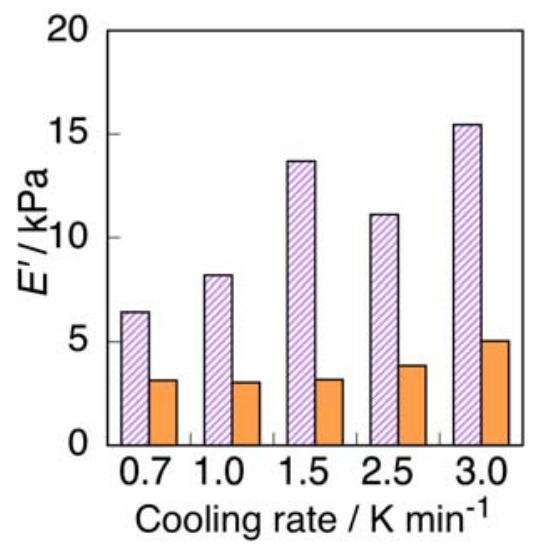

275

276

277

278

279 280

Fig. 8 Comparison of dynamic modulus ( $\left.E^{\prime}\right)$ measured by TMA of CG and LBG. Shaded bars ;LBG; solid bars ; CG.
(a) Concentration of $\mathrm{CG}$ and $\mathrm{LBG} ; 1 \%$, cooling rate; $1 \mathrm{~K} \mathrm{~min}^{-1}$
(b) Concentration of CG and LBG; 1\%, number of freezing and thawing $(n) ; 1$

\section{0}

The above facts indicate that once the cross-linking zone is formed via intermolecular association at the initial freezing process, the network structure of CG gels is maintained and no further molecular association occurs by repeating freezing. It can be concluded that the size of ice initially formed inside the gel is memorized and crystallization occurs in a similar manner by successive freezing and thawing. Cooling rate kinetically affects the aggregation of polymer chains, and therefore polymer 
concentration decreases with increasing cooling rate. Size of ice depends on cooling rate, and quick cooling forms a small size of ice which induces a dense network structure, although this effect on CG is not marked compared with that of LBG as shown in Fig. 8(b). According to computer simulations of FG, GG, Tara-G and LBG, it is thought that the galactosyl side groups have the effect of lowering the chain dimension. The intra-chain associations via H-bonding along the side groups are also ascertained [4]. The galactosyl side groups associate and form a cross-linking area or junction zone. According to $\mathrm{M} / \mathrm{G}$ ratio, the stiff chain attributed to the unsubstituted region of $\mathrm{CG}$ is longer than that of LBG. Accordingly, it is considered that once H-bonding is established, further molecular association is disturbed by stiff main chains, since the molecular chain distance between cross-linking area of CG is considerably large. The difference between LBG and CG observed in $E^{\prime}$ can be explained by mannose/galactose ratio. At the same time, it is reasonable to assume that FG, GG and Tara-G do not form hydrogels by freezing and thawing, since molecular conformation is too compact for ice crystallization which induces inter-molecular H-bonding.

\section{Conclusions}

CG hydrogels were prepared by freezing and thawing. $n$ scarcely affects gelation ability. This indicates that once the network structure is formed, molecular rearrangement does not occur. In contrast, it is clearly seen that cooling rate evidently affects gelation ability. $E$ ' values by measured TMA in water increased with increasing cooling rate. $E$ ' of CG are far lower than those of LBG. It is thought that the galactosyl side groups associate and form a cross-linking area or junction zone. The low E' value of CG gels is ascribed to the fact that association of galactosyl side group is disturbed by stiff chains attributed to the unsubstituted region of $\mathrm{CG}$

This paper was presented at the International Conference on Thermal analysis and Calorimetry 2012, held at Osaka, Japan in August 2012. This work was supported by a Grant-in-Aid for Scientific Research (Young Scientists (B)). 
322 Acknowledgements The authors extend their sincere thanks to Professor Clive S.

323 Langham, of Nihon University for his helpful comments.

\section{References}

1. Singh V, Sethi R, Tiwari A. Structure elucidation and properties of a non-ionic galactomannan derived from the Cassia pleurocarpa seeds. International Journal of Biological Macromolecules.2009;44:9-13.

2. Singh V, Srivastava A, Tiwari A. Structural elucidation, modification and characterization of seed gum from Cassia javahikai seeds: A non-traditional source of industrial gums. International Journal of Biological Macromolecules. 2009;45:293-7.

3. Funami T, Kataoka Y, Noda S, Hiroe M, Ishihara S, Asai I, Takahashi R, Inouchi N, Nishinari K. Functions of fenugreek gum with various molecular weights on the gelatinization and retrogradation behaviors of corn starch-2:Characterizations of starch and investigations of corn starch/fenugreek gum composite system at a relatively low starch concentration; 5 w/v\%. Food Hydrocolloids, 2008;22:777-87.

4. Wu Y, Li W, Cui W, Eskin N.A.M, Goff H.D. Molecular modeling approach to understand conformation functionality relationships of galactomannans with different mannose/galactose ratio. Food Hydrocolloids, 2012;26:359-64.

5. Hallagan J.B, La Du B.N, Pariza M.W, Putnam J.M, Borzelleca J.F. Assessment of cassia gum. Food and Chemical Toxicology. 1997;35:625-32.

6. Tanaka R, Hatakeyama T, Hatakeyama H. Interaction between polymer molecules in locust bean gum-water systems during cooling and freezing processes.Williams P.A. Phillips G.O, editors. Gums and Stabilisers for the Food Industry 9.Cambridge:The Royal Society of Chemistry; 1998.pp. 43-7.

7. Tanaka R, Hatakeyama T, Hatakeyama H. Formation of locust bean gum hydrogel by freezing-thawing. Polym. Int. 1998;45:118-26.

8. Hatakeyama T, Naoi S, Iijima M, Hatakayama H. Locust bean gum hydrogels formed by freezing and thawing. Macromol. Symp. 2005;224:253-62.

9. Iijima M, Hatakeyama T, Hatakeyama H. DSC and TMA studies on freezing and thawing gelation of galactomannan polysaccharide. Thermochim.Acta. 
2012;532:83-7.

10. Nagura M, Nagura M, Ishikawa H. State of water in highly elastic poly(vinyl alcohol) hydrogels prepared by repeated freezing and melting. Polymer Communications Guildford. 1984;25:313-4.

11. Nagura M, Hamano T, Ishikawa H. Structure of poly(vinyl alcohol) hydrogel prepared by repeated freezing and melting. Polymer. 1989;30:762-5.

12. Hatakeyama T, Yamauchi A, Hatakeyama H. Effect of thermal hysteresis on structural change of water restrained in poly(vinyl alcohol) pseudo-gel. Eur. Polym. J.1987;23:361-5.

13. Hatakeyama T, Uno J, Yamada C, Kishi A, Hatakeyama H. Gel-sol transition of poly(vinyl alcohol) hydrogels formed by freezing and thawing. Thermoochim. Acta. 2005;431:144-8.

14. Iijima M, Hatakeyama T, Takahashi M, Hatakeyama H. Thermomechanical analysis of polysaccharide hydrogels in water. J. Therm. Anal. Calorim. 2001;64:617-27.

15. Iijima M, Hatakeyama T, Nakamura K, Hatakeyama H. Thermomechanical analysis of calcium alginate hydrogels in water. J. Therm. Anal. Calorim. 2002;70:807-14.

16. Iijima M, Hatakeyama T, Nakamura K, Hatakeyama H. Effect of annealing on calcium pectin gel formation by thermomechanical analysis. J. Therm. Anal. Calorim. 2002;70:815-24.

17. Iijima M, Hatakeyama T, Hatakeyama H. Swelling behavior of calcium pectin hydrogels by thermomechanical analysis in water. Thermochim. Acta.2005;431:68-72. 